\title{
Multiphysics Analysis of Contact Pressure Profile of Airless Tires as compared to Conventional Tires
}

\author{
S Ludvigsen', Z Andleeb², H Khawaja ${ }^{1,4 *}$, \\ M Moatamedi ${ }^{3,4}$, B Alzahabi ${ }^{4}$
}

1. UiT-The Arctic University of Norway

2. Ghulam Ishaq Khan Institute of Engineering Sciences and Technology, Pakistan

3. Oslo Metropolitan University, Norway

4. Al Ghurair University, UAE

\begin{abstract}
The harsh climate of the Arctic has always posed significant challenges to car drivers. The severe loss in traction due to snow and icing on the roads has led to an increased risk of collisions. The chapter compares the conventional air-filled tire with a non-pneumatic tire to improve the grip in the Arctic conditions. The grip obtained for tires is determined by the weight of the car and the friction between the tire and the road. The friction coefficient, used to determine friction, is a function of the contact pressure. This chapter discuss research work to obtain a concentrated pressure profile for the airless tire, compared to a conventional tire. A finite element analysis using ANSYS ${ }^{\circledR}$ Workbench is performed on two distinct models. The different pressure profiles of the models are analyzed, and the results proved the non-pneumatic tires have a more concentrated pressure profile with higher pressure values.
\end{abstract}

\section{INTRODUCTION}

The Cold climatic conditions of the Arctic severely affects many areas of life. Accumulation of ice during winters is a common phenomenon that hinders the normal operations of various mechanical structures. Researchers are continuously proposing new ways to cope with it [1][2][3][4][5]. Cold conditions also make driving significantly harder and more unpredictable. The first winter tires used for road cars were applied during the mid-1930. The difference to these tires compared to the summer tires were the enlargement of the grooves. Deeper lateral groves gave better traction as it allowed the tread blocks to dig deeper into the snow-covered roads. In 1961 the first tires with metal studs were introduced [6]. The studs help the tire gaining a grip on hard-packed snow and ice as the studs' claws into the ice and increases friction. The focus during the last 50 years has been on improving traction by optimizing rubber compositions and treading.

${ }^{*}$ Corresponding Author: hassan.a.khawaja@uit.no 
This was done by changing parameters like elasticity, hardness, adding of studs as well as modifying tread pattern design. In recent years the government and manufacturers have been looking towards stud-free winter tires due to environmental benefits. The implementation of studs is not in practice nowadays due to the ever-increasing focus on air pollution and environmental hazards caused by road dust produced by studded tires [7][8].

\subsection{Common Definition of Grip}

The general perception of having a good grip is when two objects with forces parallel to their contact area, do not move relative to each other. In other words, the grip is the force that gives the object the ability to stay in contact with surfaces without slipping, referred to as frictional force [9]. Grip can be broadly differentiated into three categories: no grip, sliding, and sticking. No grip means no resistance against the movement. Sliding friction is having a sliding motion but with friction working against the direction of travel. This friction force will limit the velocity by transferring the kinetic energy into potential energy, in form of heating generation on the contact surface and the object. Sticking grip is where there is enough frictional force to prevent any movement between the two objects. This means, from a stationary position the frictional force is greater than the force trying to move the object. The surface friction coefficient is the surface property stating the gripping ability against each other. The friction coefficient is higher if the contact surface is rough and dry, compared to a lower friction coefficient if it is smooth and covered with a lubricant such as a grease or another slippery fluid [10]. In addition to the above, the frictional force is directly proportional to the normal force or applied pressure.

There are two factors that change the level of grip or friction: surface coefficient of friction and normal force, as illustrated in the Eq. (1).

$$
F_{\text {friction }}=\mu \cdot N
$$

where $F_{\text {friction }}$ is the frictional force, $\mu$ is the surface friction coefficient, and $N$ is the normal force as shown in Eq. (2),

$$
N=\int_{A} P d A
$$

where $\mathrm{P}$ is the pressure, and $\mathrm{A}$ is the surface area. 


\subsection{Tire Components}

The components of the tire listed below can be seen graphically in Figure 1 [11].

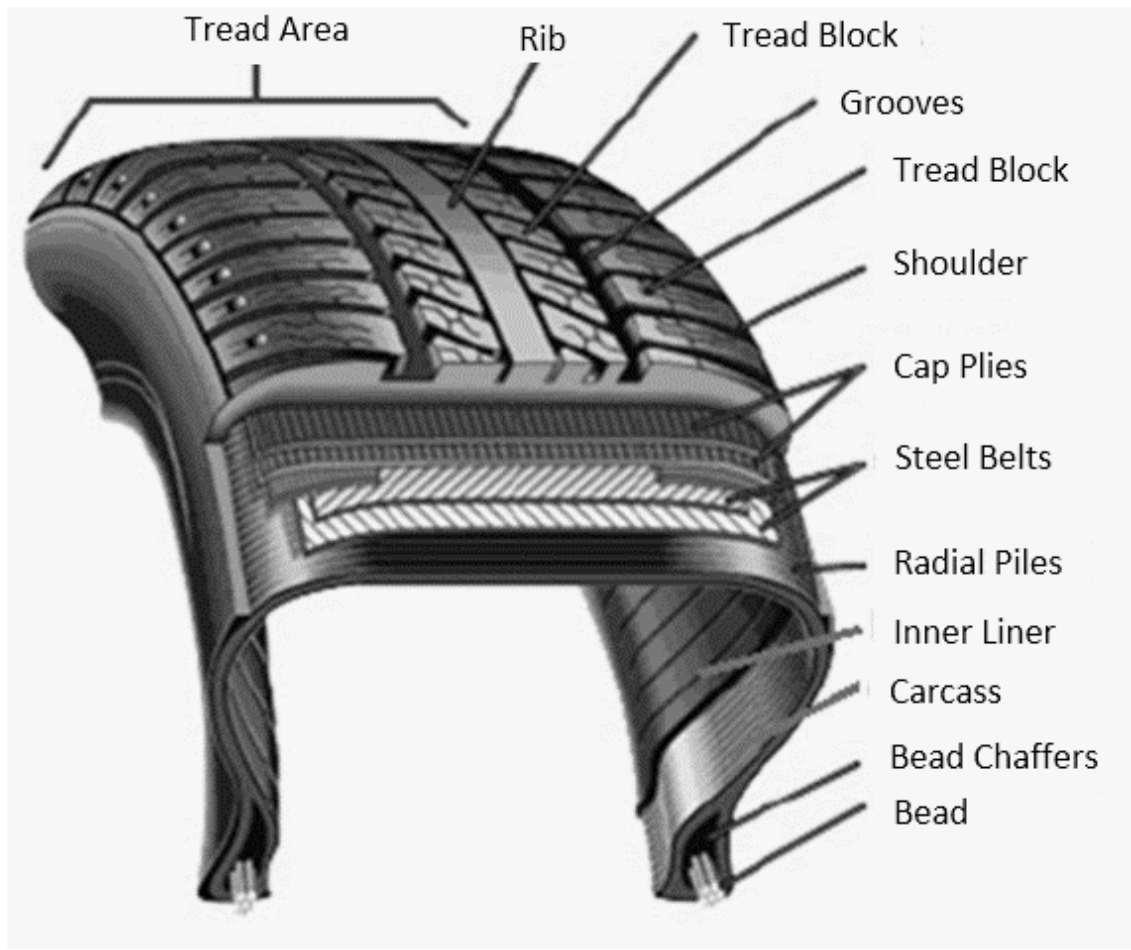

Figure 1: tire's anatomy[11]

(1) Inner liner: The first material from the inside is a synthetic rubber providing an airtight layer. It is to prevent any leakage of air, which would lead to a pressure loss inside the tire.

(2) Carcass: The carcass is made with strong textile fiber cords implemented in a rubber housing. Its objective is to maintain the tire's shape under internal pressure. It also ensures that the tire would not bulge out when inflating it.

(3) Beads: The beads are steel wires included in the part of the tire sidewall in contact with the rim. The purpose of the wires is to ensure an airtight contact between the tire and the rim to avoid any air leakage. A set of wires included in the tire can be, in some cases, withstand ten times the weight of the car [12].

(4) Sidewalls: The sidewall is where the logo of the manufacturer and the details about the tire and its production is printed. The details are preferable; dimensions of the tire, speed rating, preferred rolling direction, and the month and year its production.

(5) Steel belts: Steel belts are bounded into the rubber, providing strength. It makes the tire's ability to handle the strain from turning and preventing the tire from expanding from the centrifugal force caused by fast rotation. 
(6) Cap plies: The cap plies are rubber layers with integrated nylon that stretches around the circumference of the tire, located between the steel belts and the treading. Both layers add resistance against expansion and reduce heating induced by friction.

(7) Tread: The tread is the part of the tire in contact with the road and is the visible part from outside. It is usually monitored to determine the quality and characteristics of tire performance. The objective of the tread is to provide grip against the road surface while providing a low level of abrasion and heat generation.

(8) Grooves are the cuts in the tread, making the tread patterns. The treading is responsible for road noise mitigation, water diversion, and to provide a large contact area with a correct frictional coefficient. Grooves are explicitly designed to provide sufficient grip in given temperatures and conditions.

\subsection{Tread Differences}

There are some key differences to a winter tire compared to a summer tire other than the possible addition of studs i.e. rubber composition, presence of pores over the thickness of tire and having sipes in the tread blocks [11][13]. The most noticeable being the shape and pattern of the treading. As Figure 2 shows, the summer tire has large tread blocks divided by wide grooves. These groves are mainly oriented in the longitudinal direction (rolling direction) for maximum water displacement at higher speeds. The tread blocks are also smooth for the maximum contact area.

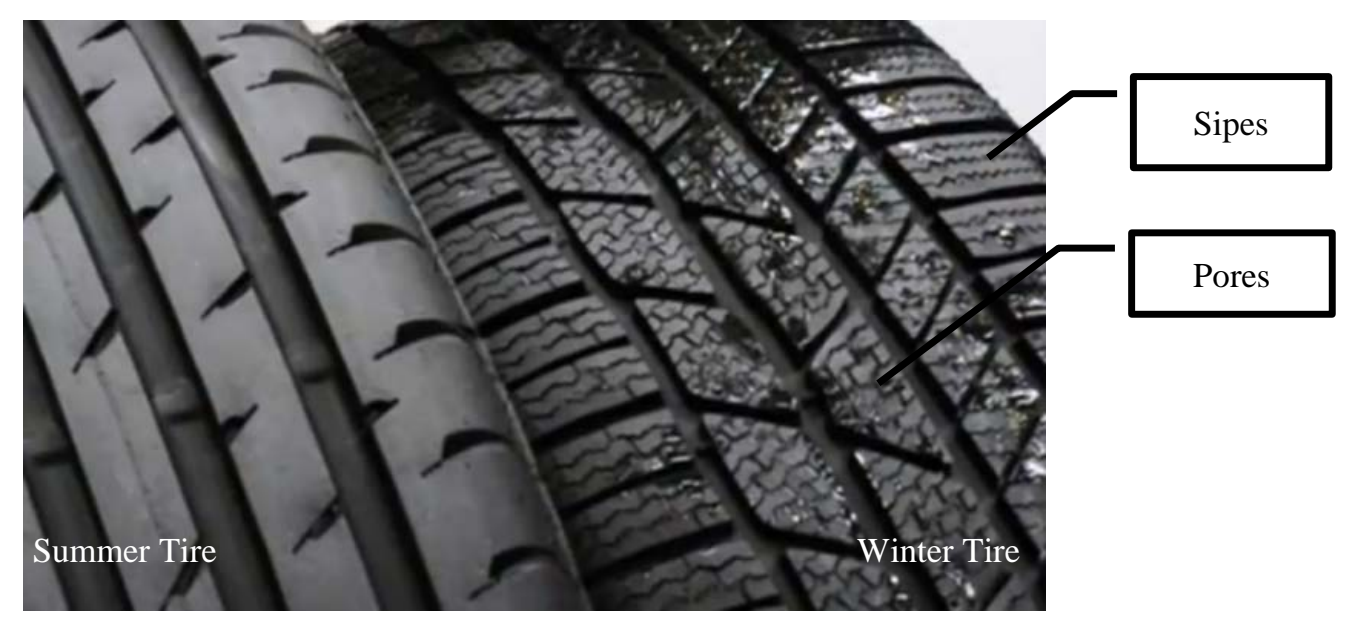

Figure 2: Comparison of summer tire vs winter tire [13].

As seen in Figure 2, the winter tire's treads have more, but narrower grooves. The grooves are a little deeper than on winter tires and oriented in different directions, including lateral movement (perpendicular to the rolling direction). It makes for an efficient deflection of water and slushy snow, providing the tread a better contact with the road without hydroplaning. It is more crucial in Arctic conditions due to more exposure to water. 
Due to the tire pressure, the snow melts due to the regelation phenomenon as the freezing point of water is lowered by applying pressure, hence creating a water film between the tires and road surface. This water needs to be moved from underneath the tread surface to prevent hydroplaning [14][15] and is effectively done by the unique tread patterns of winter tires.

\subsection{Physics of Pressure Profile}

The pressure inside a car tire can be illustrated in Figure 3. As shown pressure on every part of the surface is equal and acting perpendicular to the area. So even with a deformation of the tire due to it being pushed on the ground, the pressure is still equal throughout its inner surface.

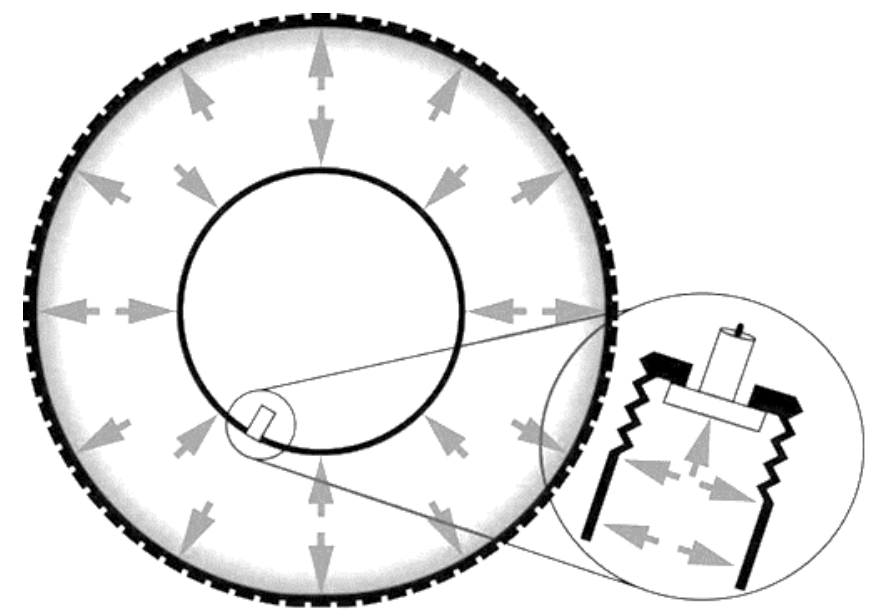

Figure 3: Illustration of uniformly distributed tire pressure [16]

correct

inflation

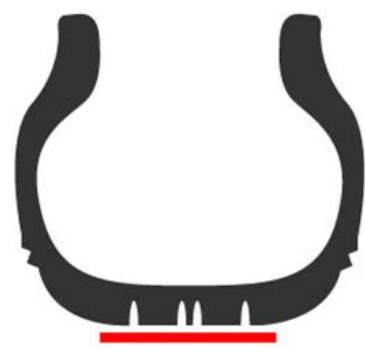

under inflation over

inflation
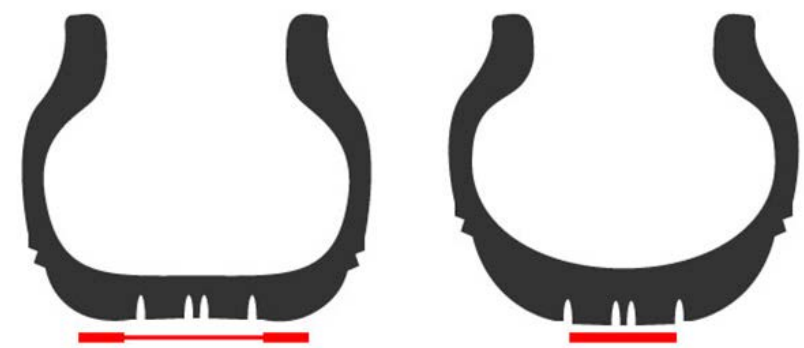

Figure 4: Illustration of tire pressure changing the contact area [21] 
This behavior is as predicted for Isotropic elastic-plastic solids at finite strain and arbitrary pressure is likely to give an equal uniform pressure profile over the contact area with the ground [17]. Some variations can occur due to the inflation pressure values, as seen in Figure 4 [18]. A drawback of using pressurized air inside the tires, especially in Arctic conditions, is that the air pressure is dependent on the temperature of the atmosphere [19][20]. This temperature effect can be neglected by changing to a non-pneumatic tire with suitable spring materials.

\subsection{Non-Pneumatic Contact Profile Theory}

The pressure profile from a non-pneumatic tire might not be the same as for pneumatic tire as we have springs transferring the weight of the car to the ground instead of air [22]. Figure 5 shows an exaggerated picture of a non-pneumatic tire being pushed down to the ground, where the red lines are symbolizing the tire springs closest to the contact area. As Hooke's law [23] explains, the force on a spring with linear behavior (constant spring constant) is determined by the displacement. It means that the springs that are being compressed the most will have the highest force. The force from these springs passes through the rubber and down to the ground, influencing the contact pressure between the tread and the road [24]. The spring being compressed the most, in a stationary situation, is always the one in the middle. This gave a higher-pressure concentration in the middle of the contact pressure profile.

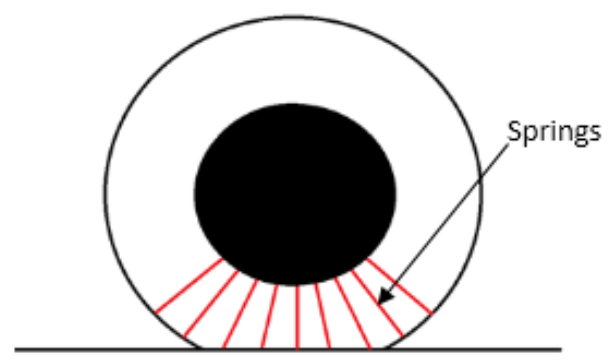

Figure 5: Illustration of the springs in a non-pneumatic tire being compressed against the road

\section{METHODOLOGY}

This work focuses on ensuring that non-pneumatic tires improve mechanical grip as compared to pneumatic tires. As conventional pneumatic tires contain pressurized air, the pressure is always uniformly distributed throughout the inside of the tire, giving it a more evenly distributed contact pressure profile as shown in Figure 6. The study was performed to change the pressure profile by concentrating the pressure from the tire down to the contact area; it was done by replacing the pressurized air with springs. This work involves a Finite Element Analysis (FEA) [25][26], CAD models were developed in Inventor ${ }^{\circledR}$, FEM analysis model was developed in ANSYS ${ }^{\circledR}$ Workbench. Pneumatic and non-pneumatic tires' results were compared. 
Similar studies have been performed in ANSYS ${ }^{\circledR}$ Workbench to analyze the materials that show the most promising results for aquaculture purposes, to simulate micro vibrations on a nontrivial torsionally oscillating structure and to optimize elastomeric micro-fluidic valve dimensions [27][28][29].

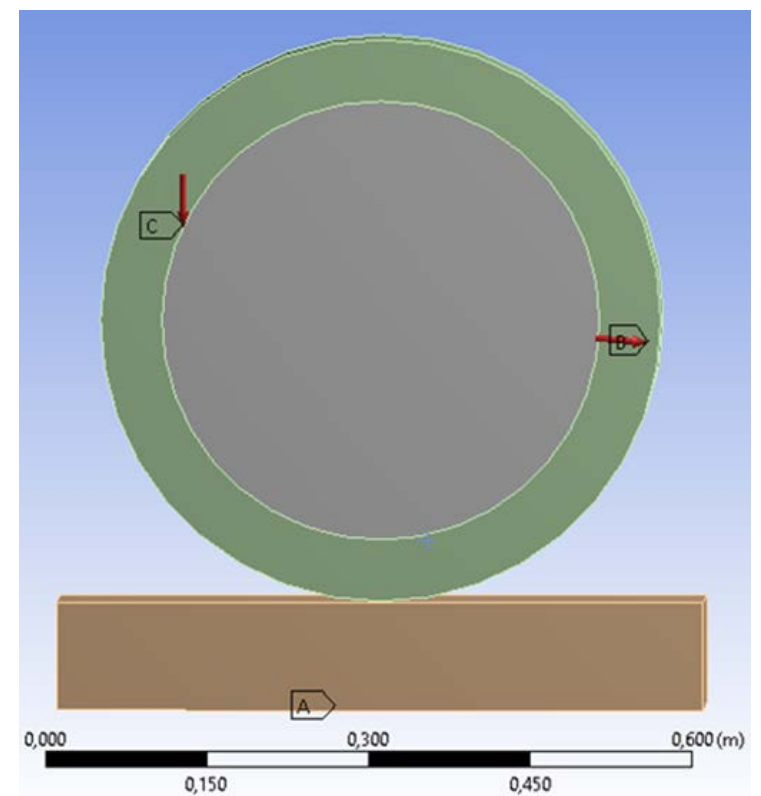

Figure 6: A schematic model displaying the fixed constraint " $A$ " at the bottom of the ground block, uniform inflation pressure "B" and applied internal pressure on pneumatic tire "C".

\subsection{Materials}

All the materials for this study were taken from the ANSYS ${ }^{\circledR}$ Workbench library. The material properties of concrete, hard and soft rubbers and custom material for springs is presented in Table 1.

Table 1: Material used for modeling in ANSYS $₫$ Workbench

\begin{tabular}{llll}
\hline Material & Volume applied to & Young's module [MPa] & Poisson ratio \\
\hline Concrete & Ground & 30,000 & 0.18 \\
Hard rubber & Pneumatic tread & 400 & 0.47 \\
Soft rubber & Pneumatic sidewalls & 10 & 0.47 \\
Custom material & $\begin{array}{l}\text { Springs for non-pneumatic } \\
\text { model }\end{array}$ & 2600 & 0.4 \\
& & \\
\hline
\end{tabular}




\subsection{Finite Element Analysis Modeling}

\subsubsection{Treading}

The idea behind the tread pattern is to make a high surface area while having grooves oriented in both longitudinal and lateral direction. The tread pattern was made in Inventor ${ }^{\circledR}$ CAD software as shown in Figure 7, the right half of the blue highlighted sketch was mirrored to create the left half of the tread but with a longitudinal offset of $10.5 \mathrm{~mm}$. With a complete drawing covering the tire's width, an extrusion of $8 \mathrm{~mm}$ was made into the tire's surface to separate the tread blocks with grooves. This extrusion was then applied a circular pattern around the tire's circumference with a number high enough to make the pattern interfere with itself again, making up a tread pattern that is the same all around. When having the right pattern, the fillet option was used to trim the edges of the tire, making realistic tire shoulders. These were made by an arched line with a radius of $8 \mathrm{~mm}$. Depth was chosen to have a smooth transition between the tread and the sidewalls.

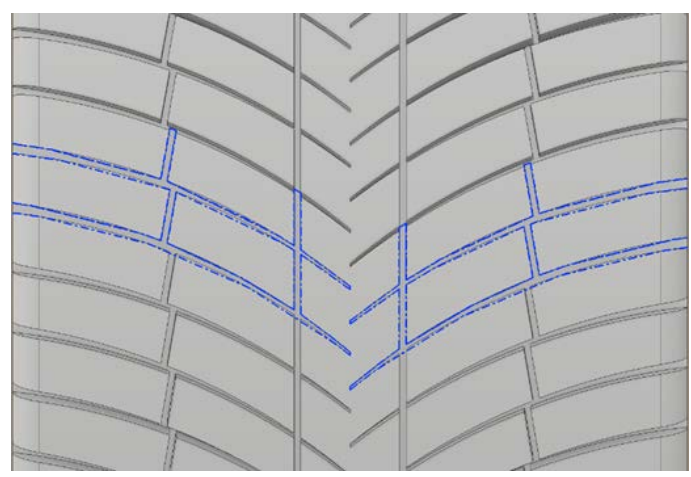

Figure 7: Tread pattern

\subsubsection{Pneumatic Tire}

The pneumatic tire model was made like a 205/55/R16 radial tire as shown in Table 2 below. As discussed in tire anatomy, there are many components embedded in the rubber of the tire. In the CAD model, only those were included that has an impact on the characteristic of a tire when applied with vertical force. The features that were developed were the sidewalls, steel belts, cap plies, carcass, and treads. The finished tire model is shown in Figure 8, and features dimensions are provided in Table 3.

Table 2: Car Tire Numbers explained

\begin{tabular}{ll}
\hline 205 & Width in millimeters \\
\hline 55 & Aspect Ratio (Section Height / Width) \\
$\mathrm{R}$ & Construction Radial \\
16 & Rim Diameter in inches \\
\hline
\end{tabular}


Table 3: Dimensions used for pneumatic tire sketch

\section{Part of tire}

The outer diameter of the tire

Tire width

Groove depth winter tire

Total rubber thickness

The radius of the rounded tire shoulder

Sidewall thickness

Sidewall height

Rim diameter

\section{Size $[\mathrm{mm}]$}

632

205

8

15

8

10

105

406

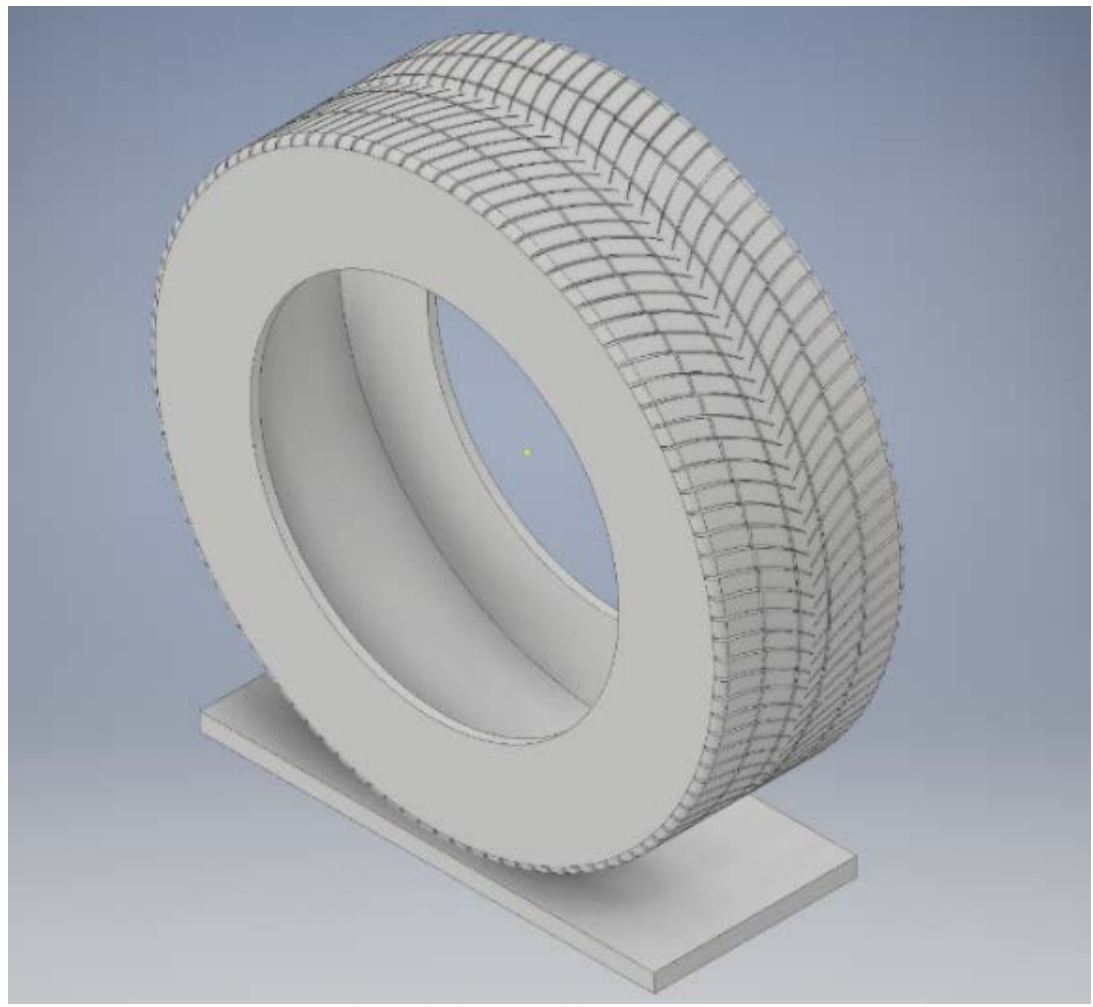

Figure 8: Pneumatic tire CAD model 


\subsubsection{Non-Pneumatic Tire}

The CAD model of the non-pneumatic tire was also made in Inventor ${ }^{\circledR}$, as shown in Figure 9. The dimensions of 205/55/R16 tire model are followed as shown in Table 2. Treads from the pneumatic model and features inside the rubber's inner circumference were redesigned. It included the removal of the sidewalls, carcass, steel belt, and cap plies from the pneumatic tire. Rim was made, represented by a solid cylinder in the center. Between the rim and the rubber, curved plates were added to act as springs, these curves had an angle of 136 degrees and a thickness of $2 \mathrm{~mm}$, making a total number of 120 springs. Dimensions of the features are shown in Table 4.

Table 4: Dimensions of non-pneumatic sketch

\begin{tabular}{ll}
\hline Part of tire & Size $[\mathbf{m m}]$ \\
\hline Total Diameter & 632 \\
Tire width & 205 \\
Rim diameter & 330 \\
Ring thickness connecting springs and rubber & 2 \\
Height of spring area & 134 \\
Groove depth & 8 \\
Total rubber thickness & 15 \\
\hline
\end{tabular}

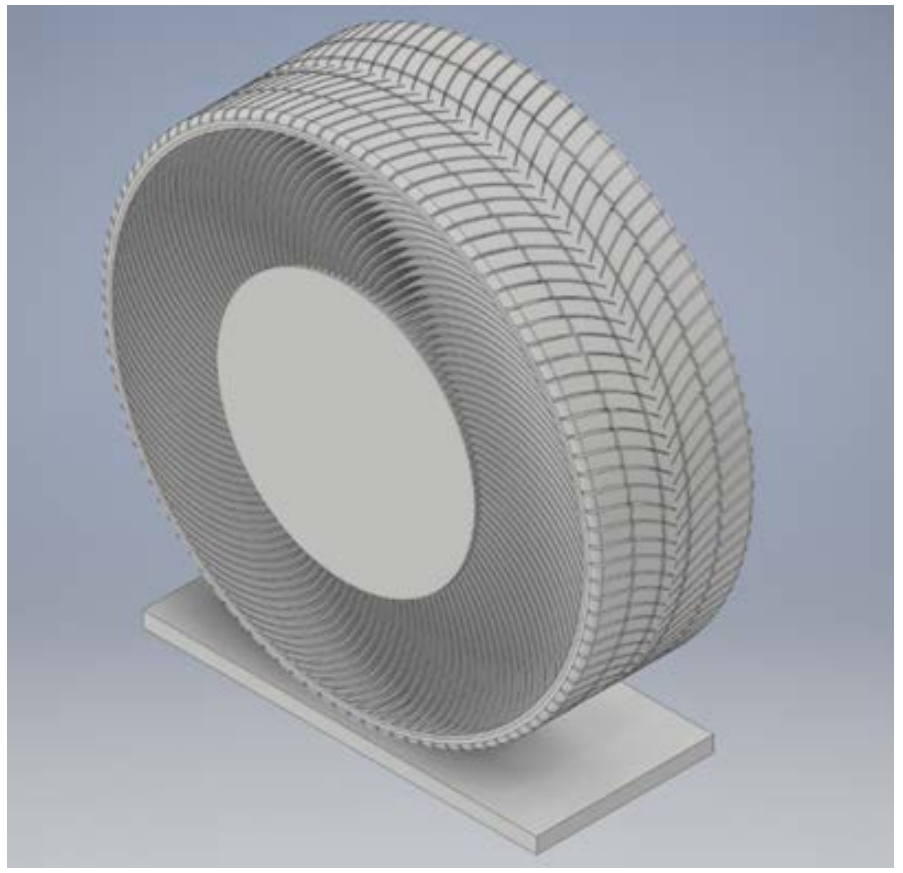

Figure 9: Non-pneumatic tire CAD model 


\subsection{Meshing}

After importing the CAD model into ANSYS ${ }^{\circledR}$ and defining the materials, meshing is done. The refinement was set to 1 , which means that each element is divided one time over both in the $\mathrm{X}$-axis and $\mathrm{Y}$-axis. By doing this, the number of elements was increased four times in the defined area. Figure 10 shows how the tread was meshed, as the tread had the same mesh for both models. The structure of the elements gave tetrahedral elements on the tire and quadrilaterals for the ground block.

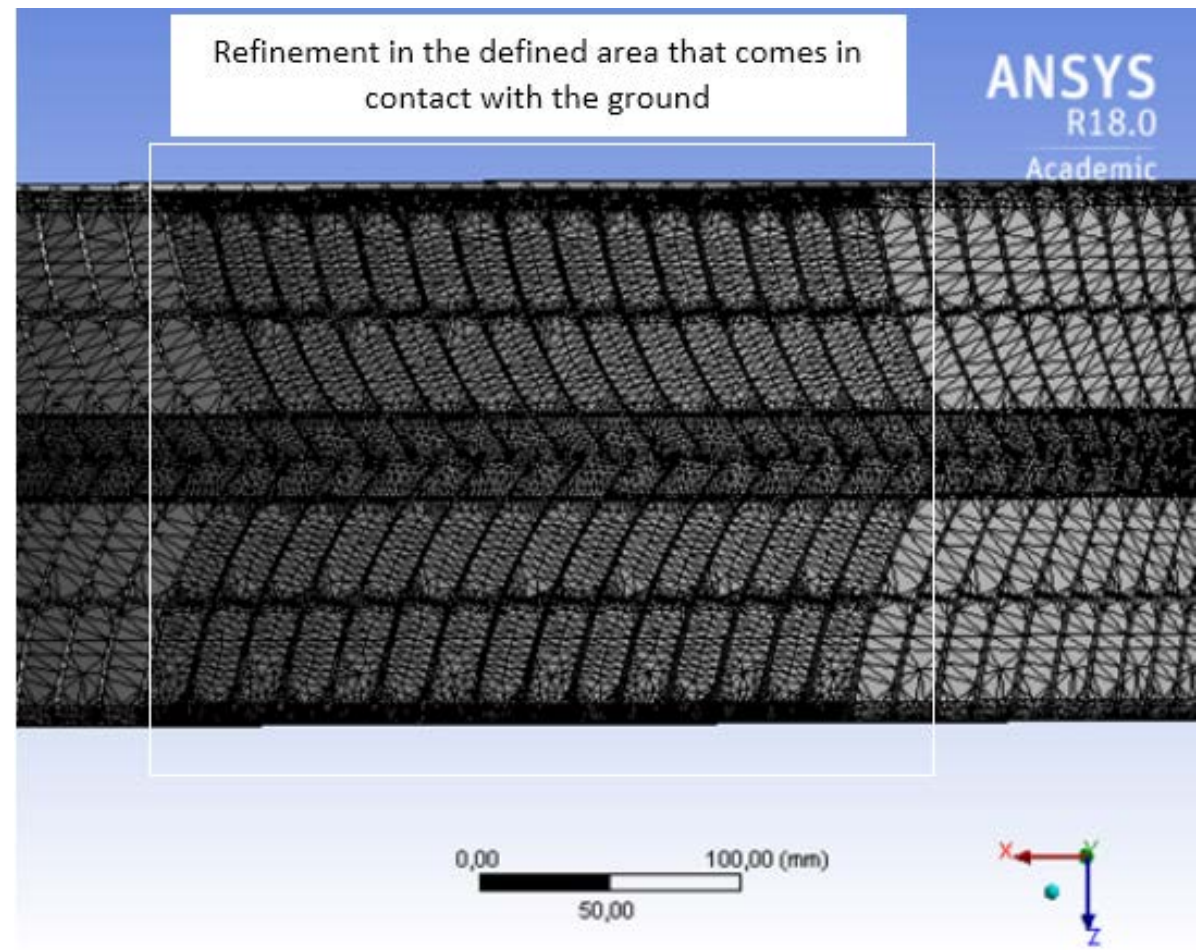

Figure 10: Meshing of the tread on pneumatic and non-pneumatic model

\subsubsection{Mesh Sensitivity Analysis}

The mesh sensitivity analysis was performed on the non-pneumatic model. Two size parameters were changed when performing this analysis; the relevance center and the span angle center, at the levels; coarse, medium, and fine. The mesh element type was set to automatic, mainly generating tetrahedral elements. The mesh sensitivity analysis graphs are presented in Figure 11. The mesh sensitivity analysis was performed on equivalent stress (Figure 11(a)), force reaction (Figure 11(b)), structural error (Figure 11(c)), and strain energy (Figure 11(d)). 


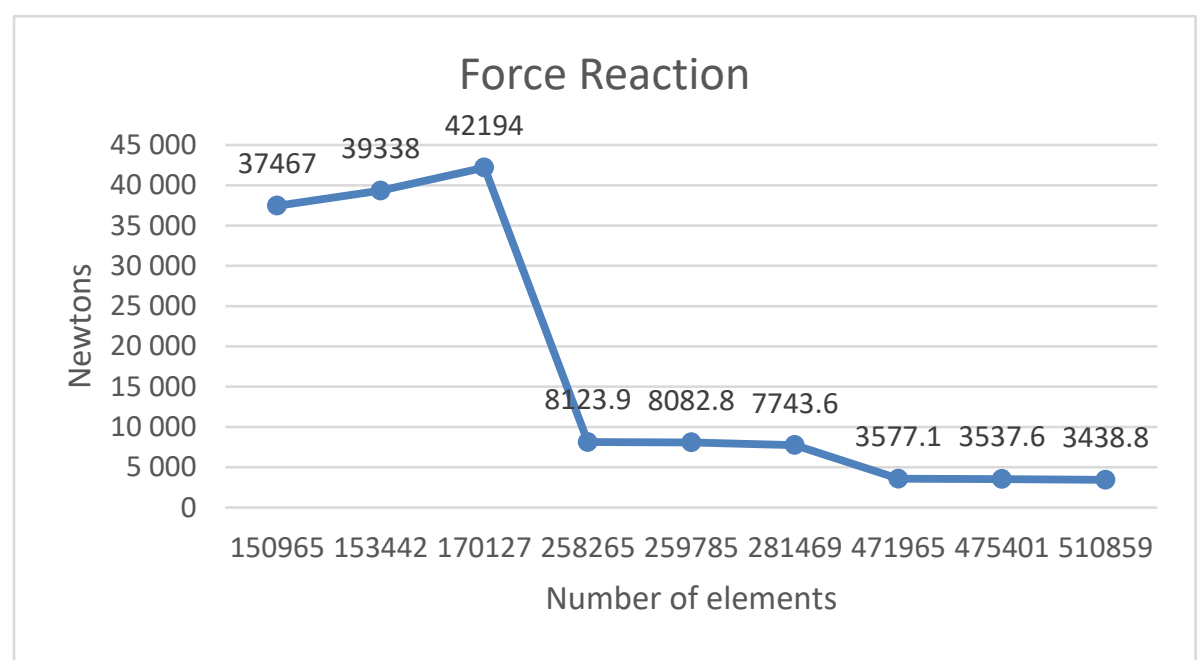

(a)

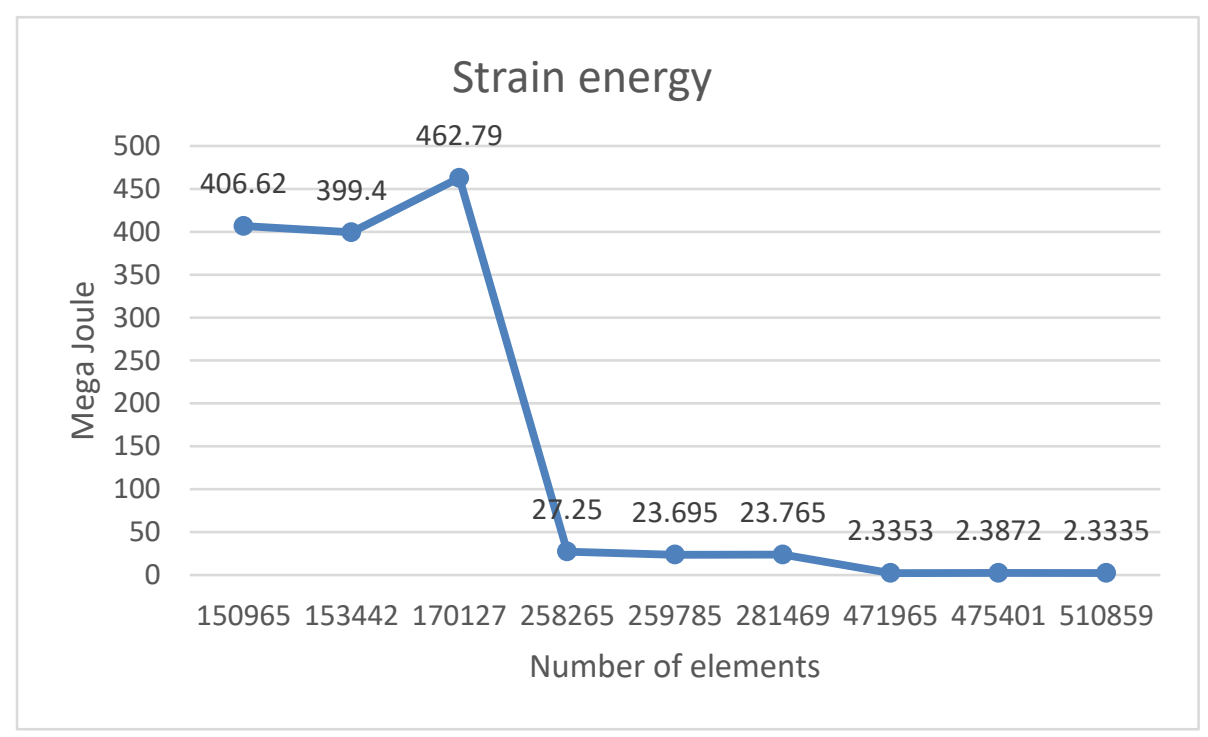

(b) 


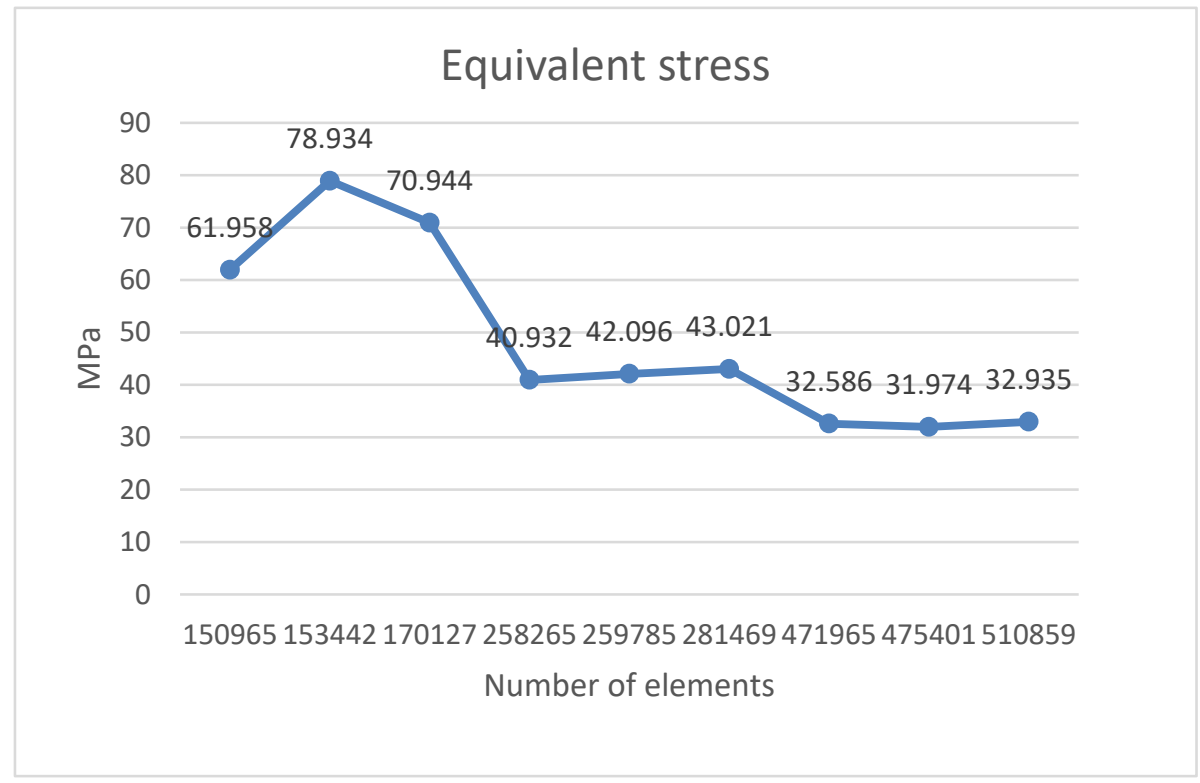

(c)

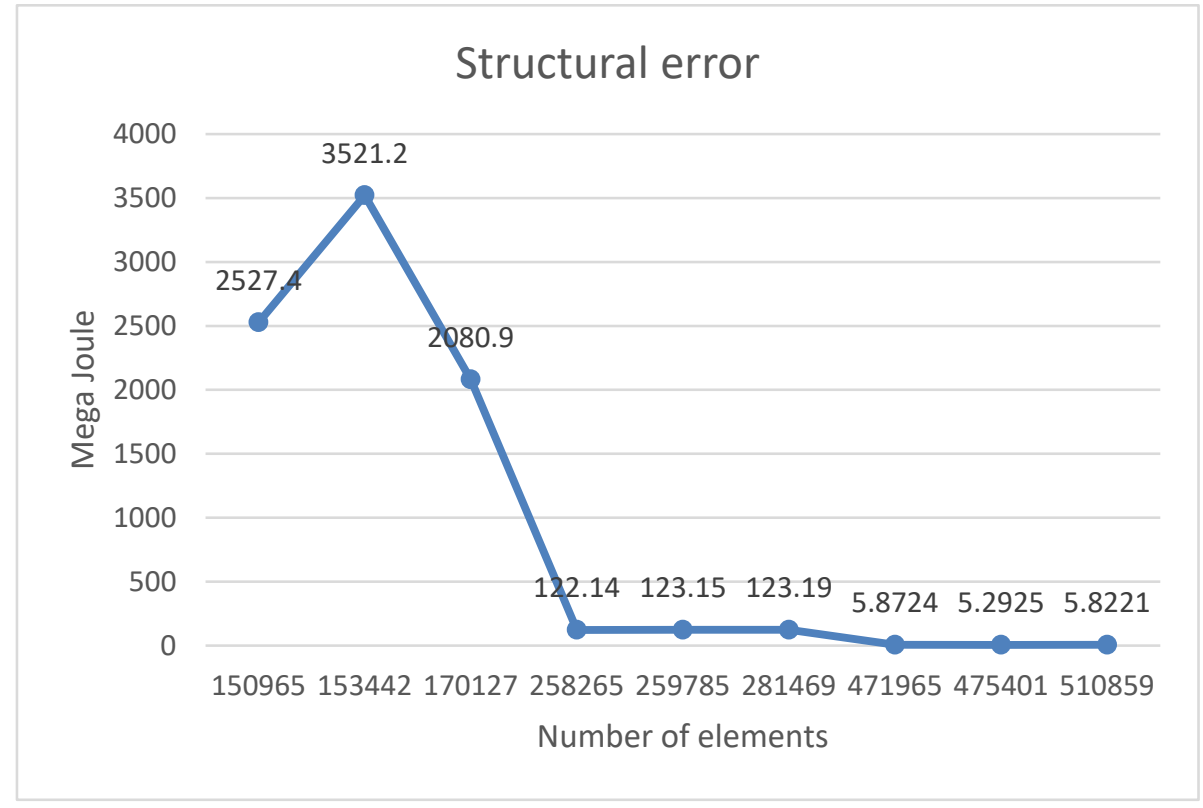

(d)

Figure 11 Mesh sensitivity analysis graphs 
This analysis shows a significant difference in result values over the different mesh densities. The relevance center is the most significant factor, compared to the span angle center, as it determines the sizing of the elements. When increasing the relevance center one level (e.g., coarse to medium), the number of elements and nodes almost doubles, while increasing the span angle center gives an increase in element number of around $10 \%$. It is clear that the coarse meshes fluctuate at high levels, while medium and fine mesh converge better. These mesh settings were applied when solving the simulations in ANSYS ${ }^{\circledR}$ Workbench.

\subsection{Boundary Condition \& Body Contacts}

For both models, the concrete block was constrained at the bottom, to prevent it from moving under pressure. The pressure from the weight of the car, calculated for each model, were applied to the outer circumference area of each rim, acting downwards.

\subsubsection{Pneumatic Model}

The contact between the tread and the sidewall were set to be 'bonded contact'. It was done to ensure that there was no material or pneumatic pressure leakage through the model under applied pressure and strain. The contact between the tread and the ground was set to frictional contact. A fixed constraint was applied to the top of the sidewall to prevent the pneumatic tire from moving. As the tire was fixed, the external force had to come from the ground.

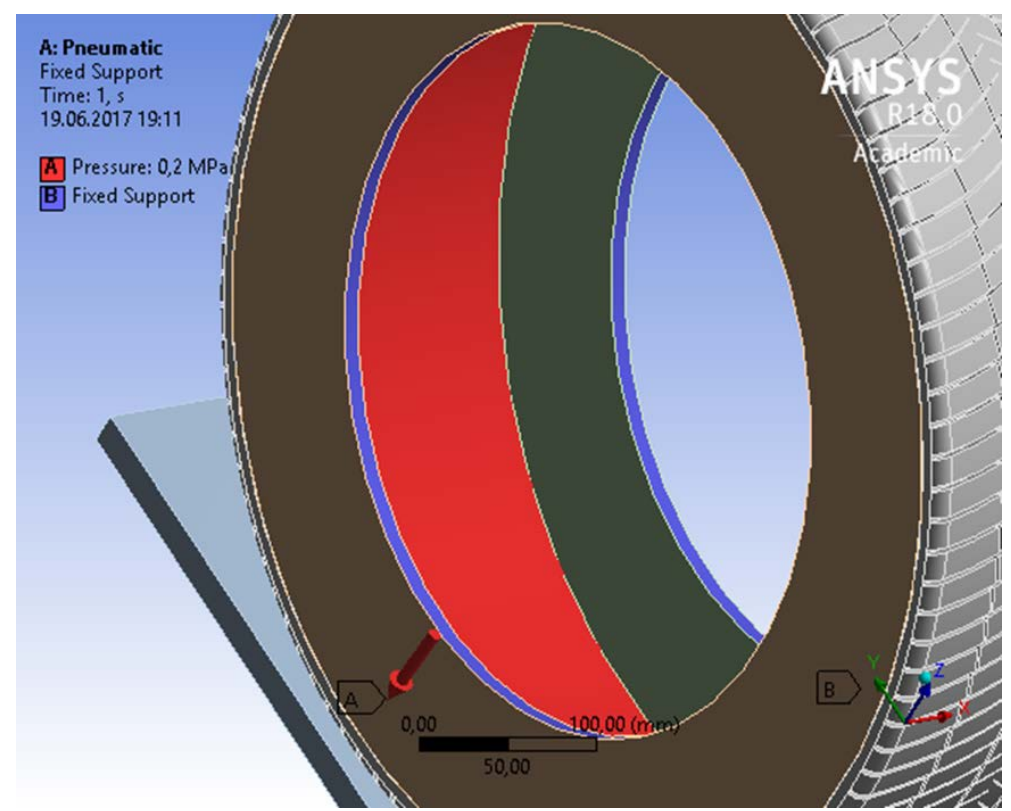

Figure 12: Fixed support and internal pressure applied to the pneumatic model 


\subsubsection{Force and Displacement}

For the tire to maintain its structure, a pneumatic pressure had to be applied. A pressure of 0.2 $\mathrm{MPa}$, or 2 bars, was applied to the inner part of the rubber acting outwards (as seen in red in Figure 12). A 2-bar pressure was used as this pressure value is representative for a 205/55R16 tire with a car weighing around 1200 kilograms. With both fixed support and a force replacing air pressure, the ground plate was moved towards the tire, aiming for a reaction force equal to $1 / 4$ of a car's weight. The reaction force is calculated by ANSYS ${ }^{\circledR}$ Workbench from the displaced body. A vehicle with balanced weight distribution on each wheel, consist of reaction force per wheel $=2974.6 \mathrm{~N}$ where the mass on each wheel $=\frac{2974.6 \mathrm{~N}}{9.81 \mathrm{~m} / \mathrm{s}^{2}}=303.4 \mathrm{~kg}$. So total mass of the vehicle $=1213.6 \mathrm{~kg}$.

\subsubsection{Non-Pneumatic Model}

The non-pneumatic model was structurally more complex than the pneumatic model because of the presence of more number of bodies, i.e., springs. There were a total of 120 springs in this model as shown in Figure 13. Before importing the CAD model to ANSYS ${ }^{\circledR}$ workbench, the bodies had to be defined in order to specify the material later. The rim, springs, and a ring around the outer circumference of the springs, were combined into one body. It left the model with three bodies: the springs, rubber tread, and the ground block.

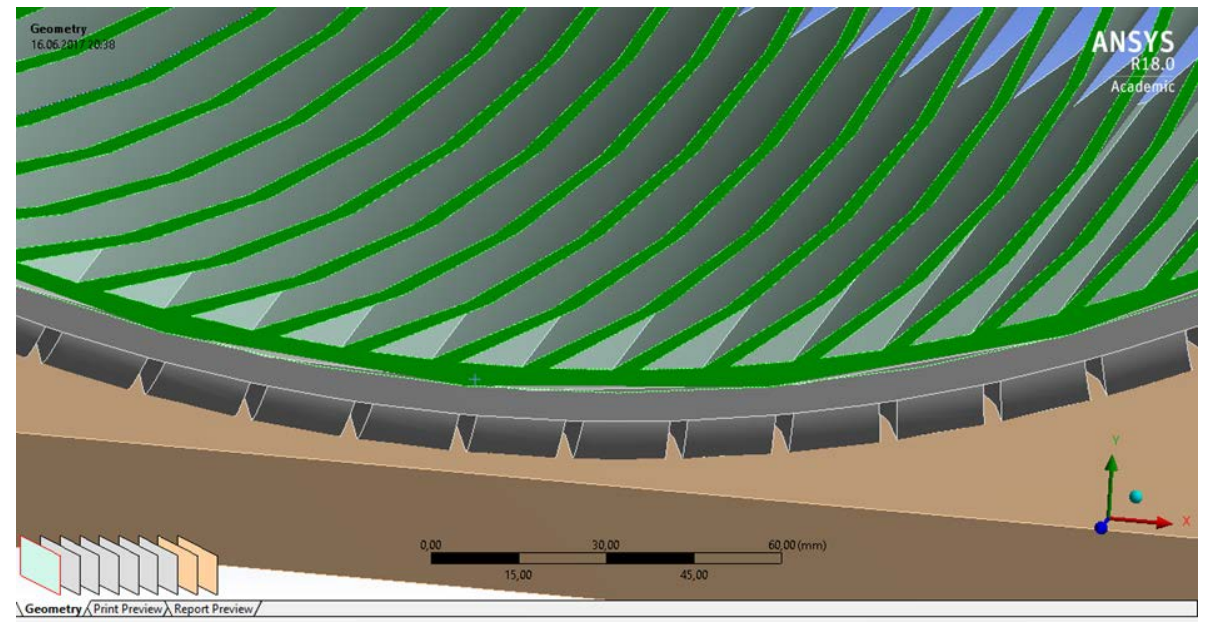

Figure 13: Springs and connected ring merged to one body

Similar to the pneumatic model, the tire was constrained. The rim surface was applied a 'fixed support', highlighted in blue in Figure 14, to ensure correct behavior and zero movements under strain from the ground plate. The springs and the rubber were 'bonded contacts' to prevent relative movement between the two. The last contact between the tread and the ground was again set to be a 'frictional contact'. 


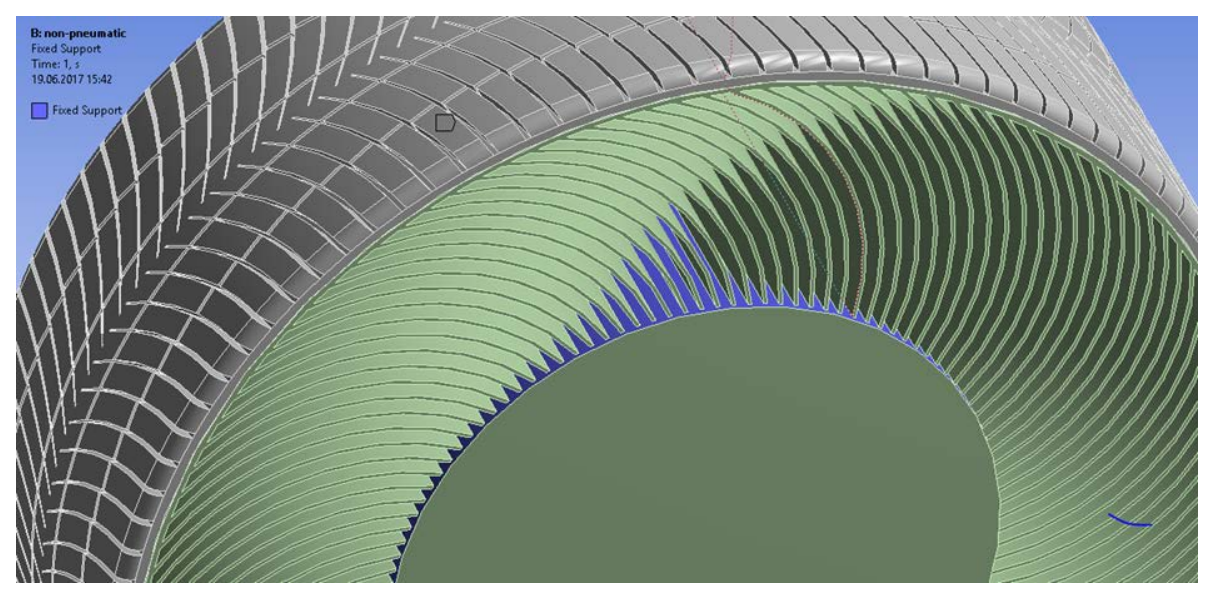

Figure 14: Fixed support, highlighted blue, added to the non-pneumatic model

\subsubsection{Force and Displacement}

The force in this model is defined as a displacement of the ground. The level of displacement was set to match the total deformation of the pneumatic model. As the pneumatic model had deformation caused by both internal force and external displacement, the total deformation was $7.9 \mathrm{~mm}$. By having the displacement determined, the aim was to get a reaction force close to the pneumatic model of $2979.3 \mathrm{~N}$. As all inputs were the same between the models, the Young's modulus (value of stiffness) for the springs were the only parameter separating the two models. After optimizing the Young's modulus, the reaction force was found to be 2976.6 Newton. It was only $3.3 \mathrm{~N}$ different from the pneumatic model, $2976.6 \mathrm{~N}$ divided by the gravity of $9.81 \mathrm{~m} / \mathrm{s}^{2}$ gives a weight of $303.7 \mathrm{~kg}$. Multiplied by the 4 tires gives a total car mass of $1214.8 \mathrm{~kg}$. It is only $0.01 \%$ different from the pneumatic model; this confirms the validity of the numerical model.

\section{RESULTS AND DISCUSSION}

3.1 Pneumatic Model

\subsubsection{Deformation}

The deformation is affected by two external factors; the pressure applied from the inside, simulating inflation pressure, and the strain from the plate pushing the tyre surface radially inwards as shown in Figure 15. The rubber characteristics worked adequately, \{ensuring that the tyre radius at the contact patch was less than the free radius around the rest of the unloaded circumference\}. The total deformation under the additional stress from the ground plate's displacement looks realistic. The highest deformations happen close to the contact area with a maximum deformation of $7.9 \mathrm{~mm}$ located at about $20 \mathrm{~cm}$ to both sides of the contact, shown in red in Figure 15. The smooth transitions between the colors indicates that the solution has converged fully and has a satisfying mesh density. 


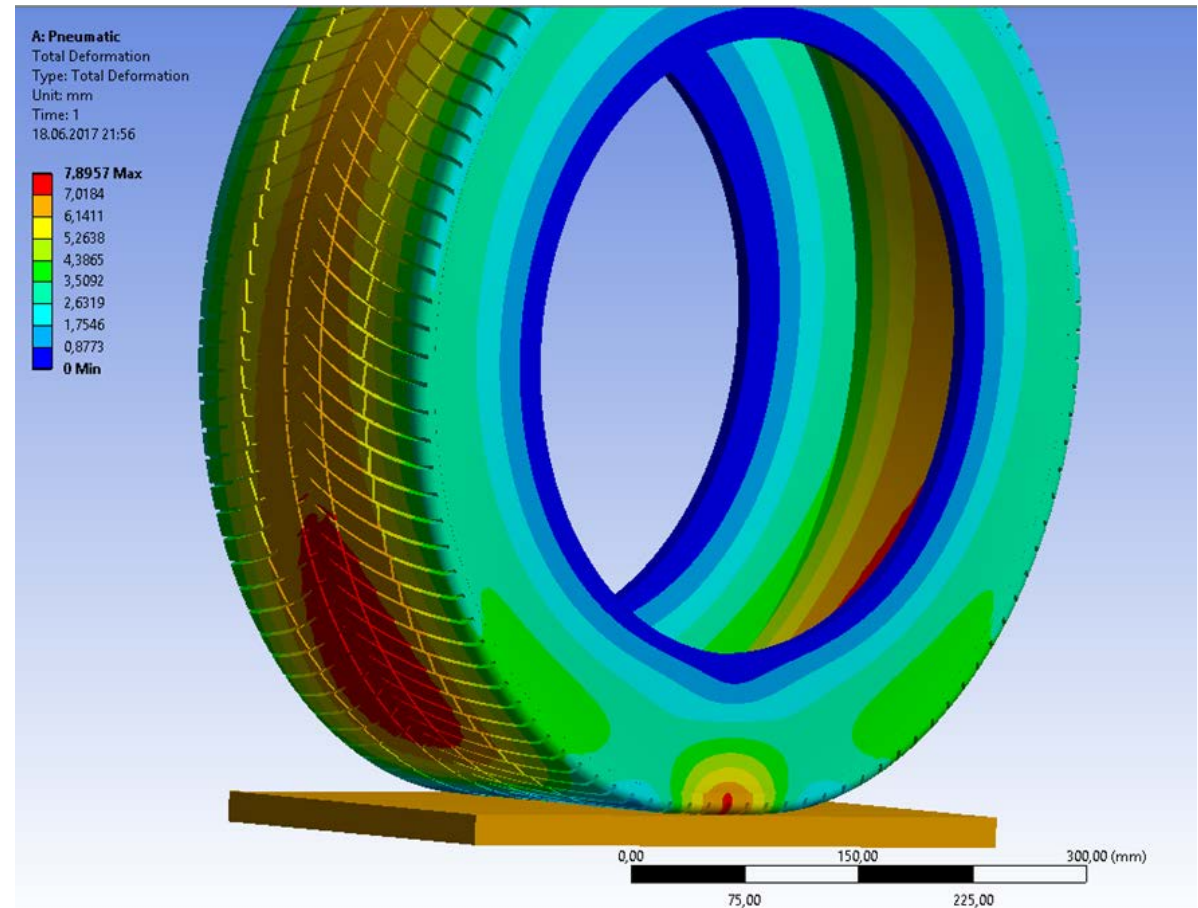

Figure 15: Deformation of pneumatic model

\subsubsection{Pressure Profile}

The pressure profile is given in Figure 16; the smoothness of the figure indicates the convergence of results. Pressure distribution through the contact area between the tire and the ground can be seen. The tire is experiencing low-pressure values in the blue zone, increasing towards the red. The average pressure over the middle area is not very high at pressures below 1.2 MPa. Two areas experience a pressure above two MPa, and that is on both sides of the tire, just below where the sidewall ends. Low tire pressure can give better traction on the loose surface such as snow and gravel due to the increase in the contact area and the tire's ability to deform over the surface unevenness. However, it is not recommended to have a too low pressure for everyday driving as the wear on edges increases and heat generations happens quicker with low tire pressure, leading to a risk of the tire cracking [30][31]. 


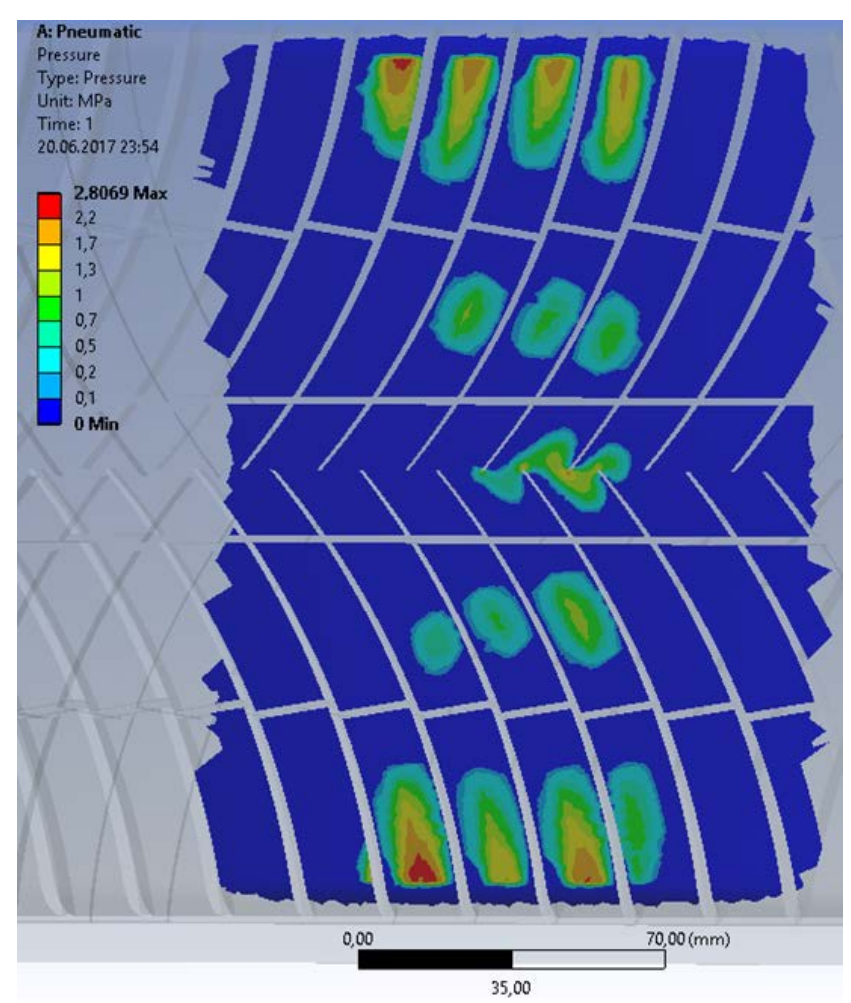

Figure 16: Pressure profile for pneumatic model

\subsubsection{Equivalent Stress (Von-Mises stress)}

The equivalent stresses are displayed in Figure 17 and Figure18. This can be interpreted that some of the high stresses are located inside the tire over the contact area, with a maximum value of $12 \mathrm{MPa}$. The other regions experiencing high pressures are in the grooves of the treading [32] which have been offset to the contact area. This is the area where the max stress of 31 MPa occurs. Another thing to notice is that the stresses are generally higher in the center of the width than on the tire shoulders. The stresses on the pneumatic model are a result of the internal and external forces. If the model runs without including the ground plate, the stress would be distributed evenly at given width over the tire's circumference because of the pneumatic pressure. Khawaja, H. A., et al. performed similar work to discuss 2-D approximation technique for solving stress analyses in FEM [33] and the Study of CRFP Shell Structures under Dynamic Loading in Shock Tube Setup [34], his work proved materials i.e. CFRP behave linearly under dynamic loading. 


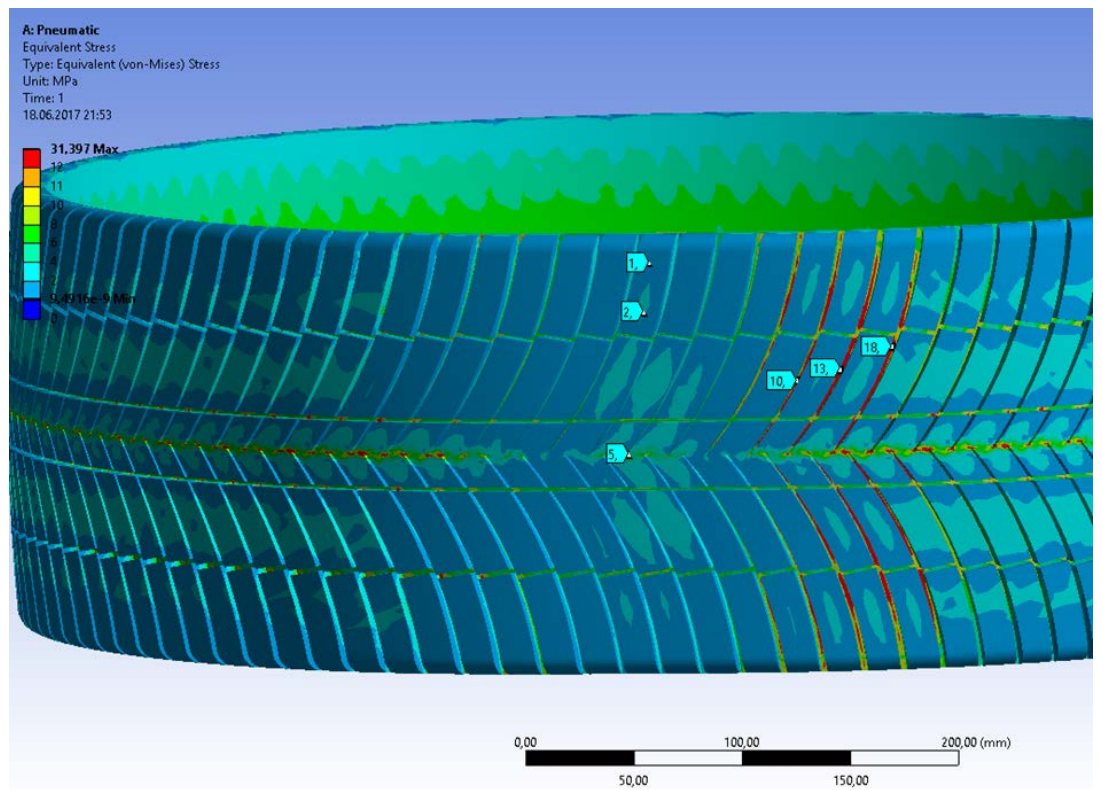

Figure 17: Equivalent stress on the outside of the pneumatic tire

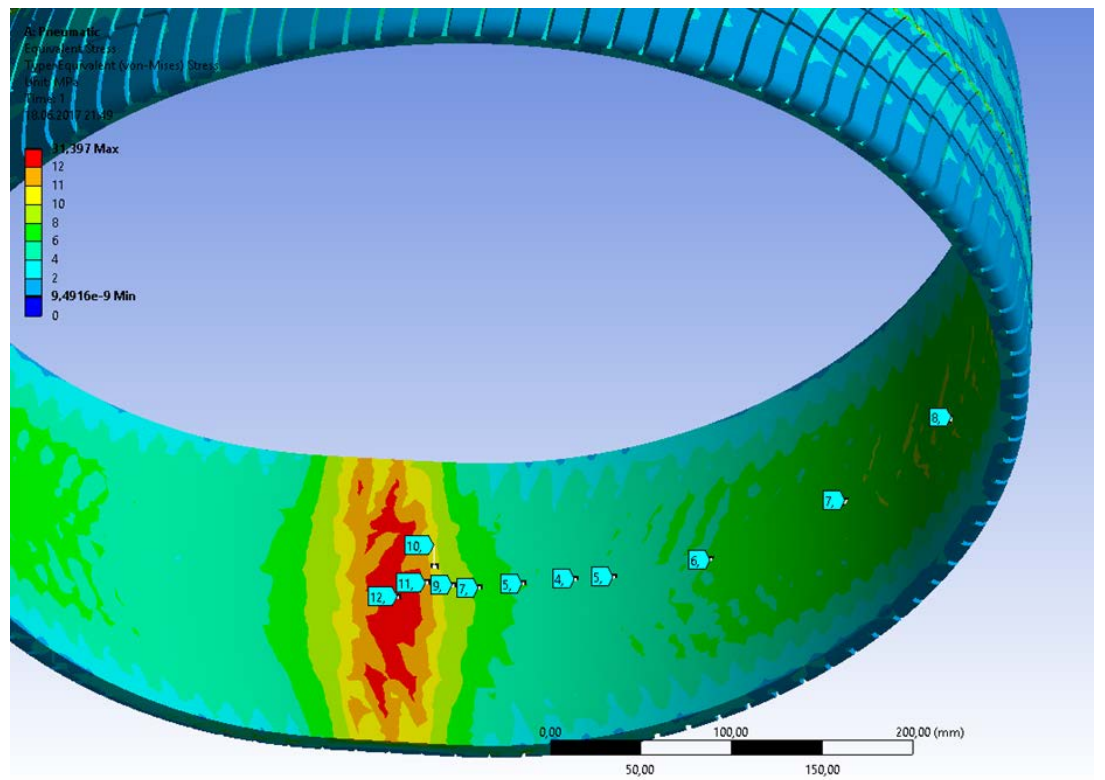

Figure18: Equivalent stress on the inside of the pneumatic model 


\subsection{Non-Pneumatic Model}

\subsubsection{Deformation}

The deformation for the non-pneumatic model is shown in Figure 19. The plate's displacement was set to $7.9 \mathrm{~mm}$ to match the total deformation of the non-pneumatic model. The highest deformation happens at the springs that are connected over the contact area. Even with deformation of $8.7 \mathrm{~mm}$, the springs still have proper clearance between them. The deformations of the model were as expected when the design was made. The springs behaved predictably, just as visualized when sketching the model. The most considerable deformation happened in the middle of the spring connected to the rubber above the contact area. This deformation was more significant than the displacement of the ground plate. It indicates the modeling accuracy of the non-pneumatic tire model.

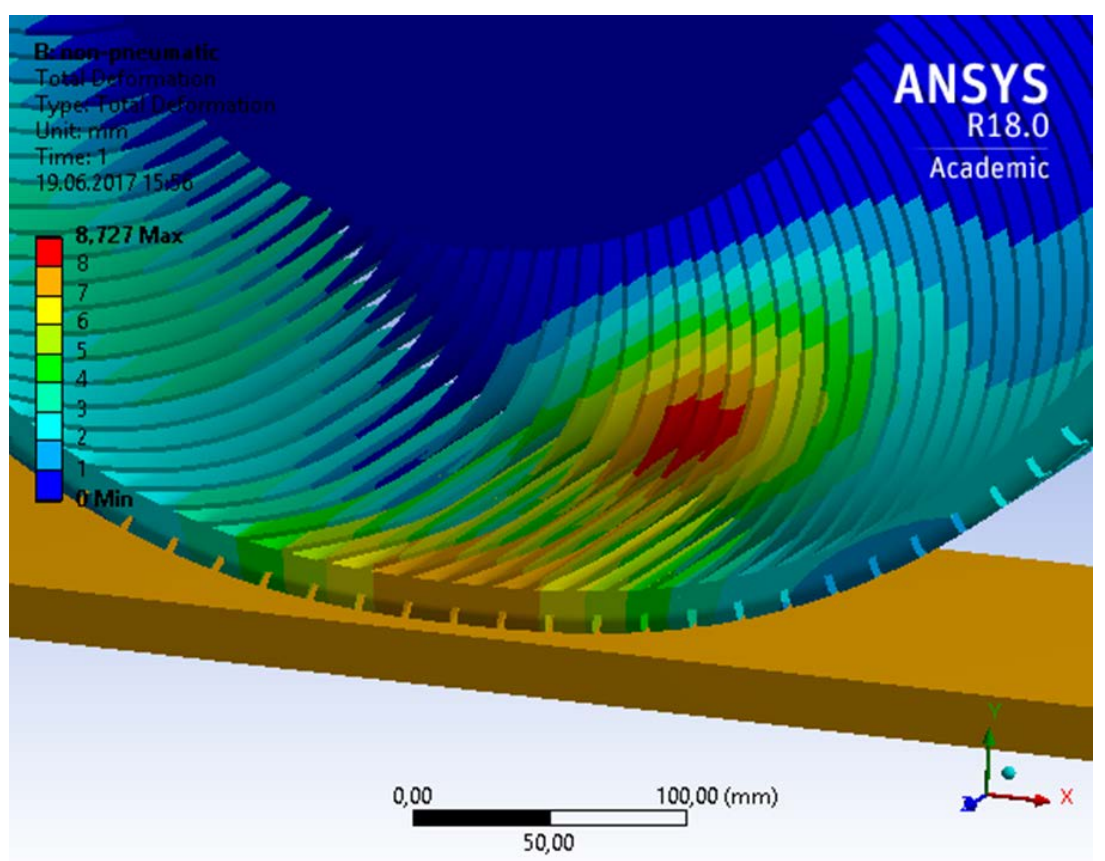

Figure 19: Deformation of non-pneumatic model

\subsubsection{Pressure Profile}

Figure 20 shows the pressure profile for the non-pneumatic model. Again, the figure is smooth and has no abnormalities in the pressure distribution. This indicates that the meshing used was of a sufficient density. For the most part, the pressure is uniformly distributed over the width of the tire and the contact area. The pressures are above 1.6 MPa on every tread block over that width, which results in pressure concentration through the center of the contact area. The maximum pressure of $4.94 \mathrm{MPa}$ occurs in the center of the profile. 


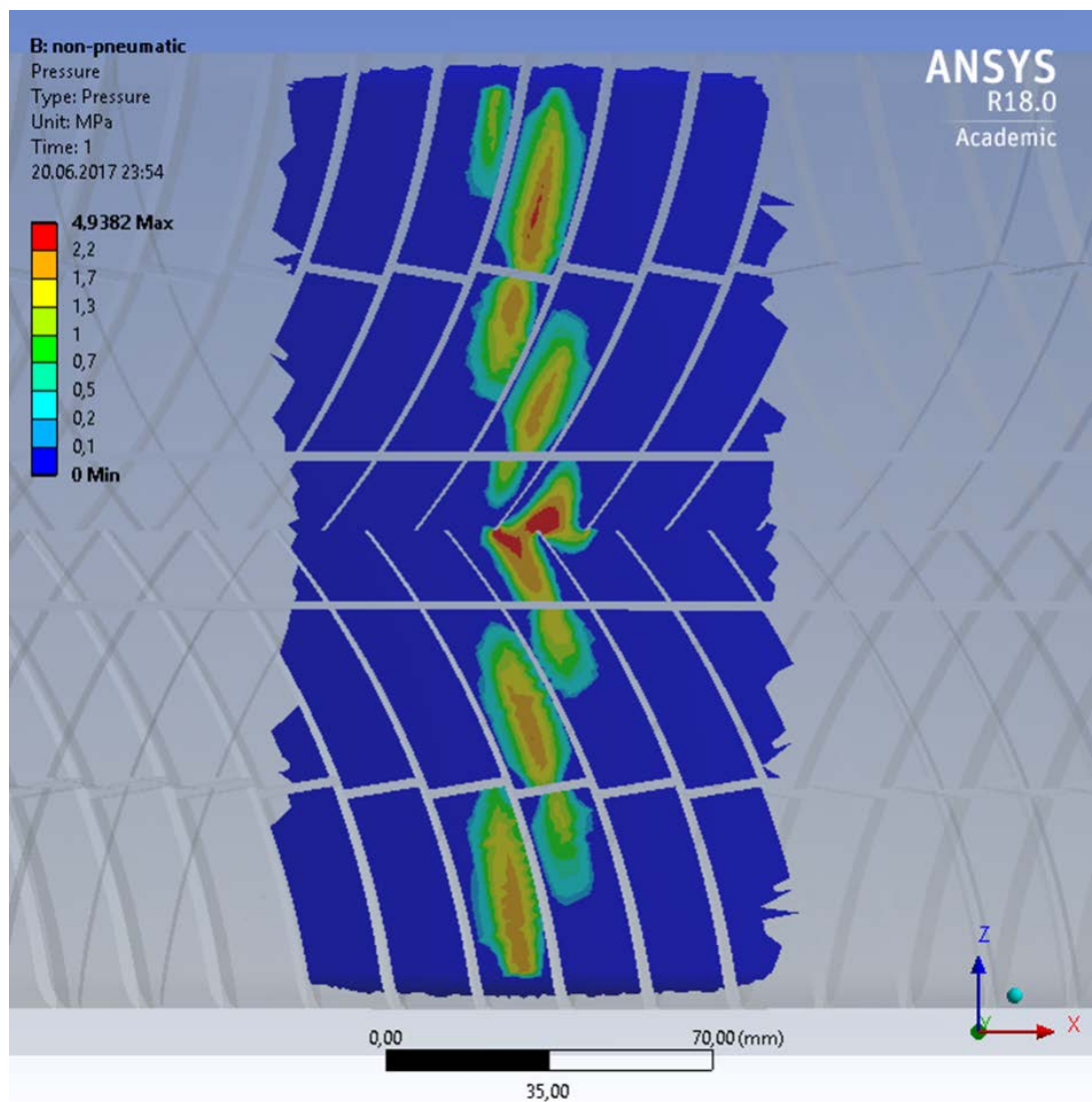

Figure 20: Pressure profile for the non-pneumatic model showing high pressure concentration at the contact area

\subsubsection{Equivalent Stress}

The equivalent stress is displayed in Figure 21. The labels show the stresses are higher at points where the springs are attached to the rim and the rubber compared to the center of the individual spring. The maximum stress appears inside the rubber in the center of the contact area. This is the same point that had both the maximum deformation and maximum pressure. Stange et al. performed similar work to visualize stresses in steel samples using IR Thermography [35][36]. 


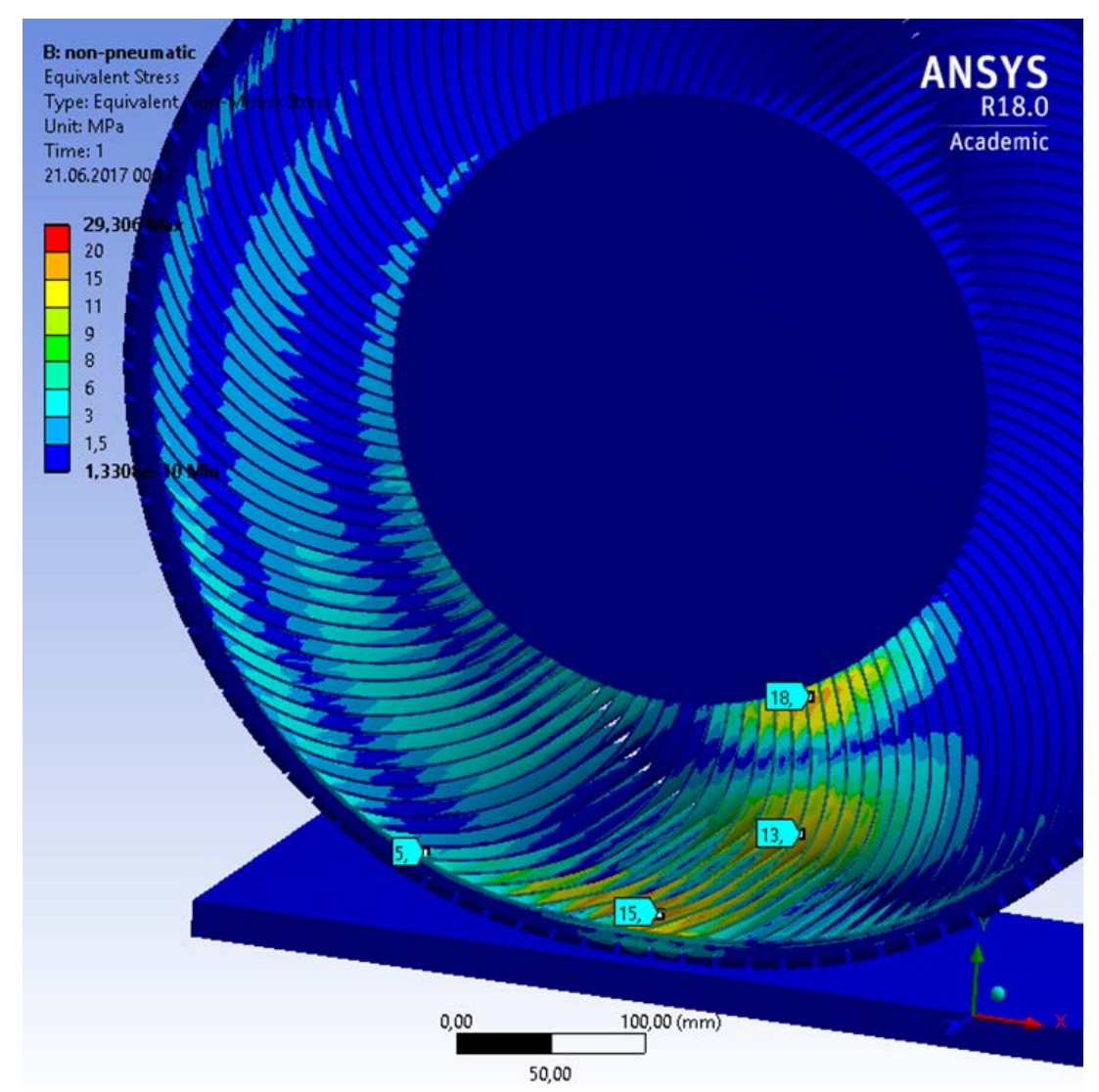

Figure 21: Equivalent stress for non-pneumatic model

\subsection{Comparison of Pressure Profiles}

When comparing the two-pressure profiles side-by-side, as shown in Figure 22, we can note the pneumatic model's contact area is larger than the non-pneumatic model. The difference is visible as the pneumatic profile has eight blocks in contact with the road than the nonpneumatic model's 6 blocks when counting in the longitudinal direction (left to right).

By analyzing the pressure profile curves as shown in Figure 23 (a) and (b), for pressure values across the mid-section of the tires, there is a clear trend. The pressure of the nonpneumatic model on the left is much more concentrated towards the center and has higherpressure values. The pneumatic model's pressure concentrations cover the longitudinal length to a more considerable extent than the non-pneumatic model, with approximately four tread blocks compared to about 2 . This is a positive result as the aim of this work is to prove that the non-pneumatic tire has a more concentrated pressure profile with higher-pressure values. 

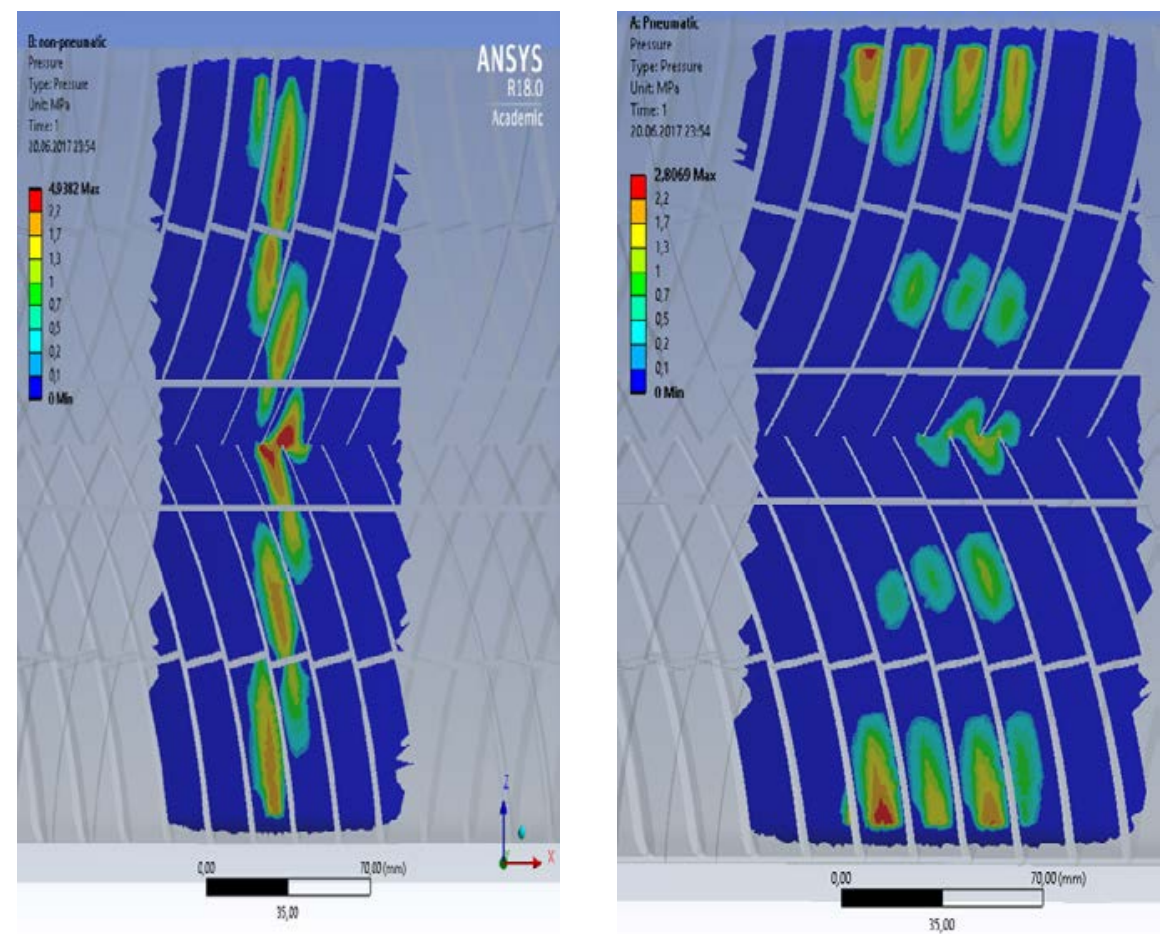

Figure 22: Side-by-side, comparison of the pressure profiles. Nonpneumatic on the left and pneumatic on the right.

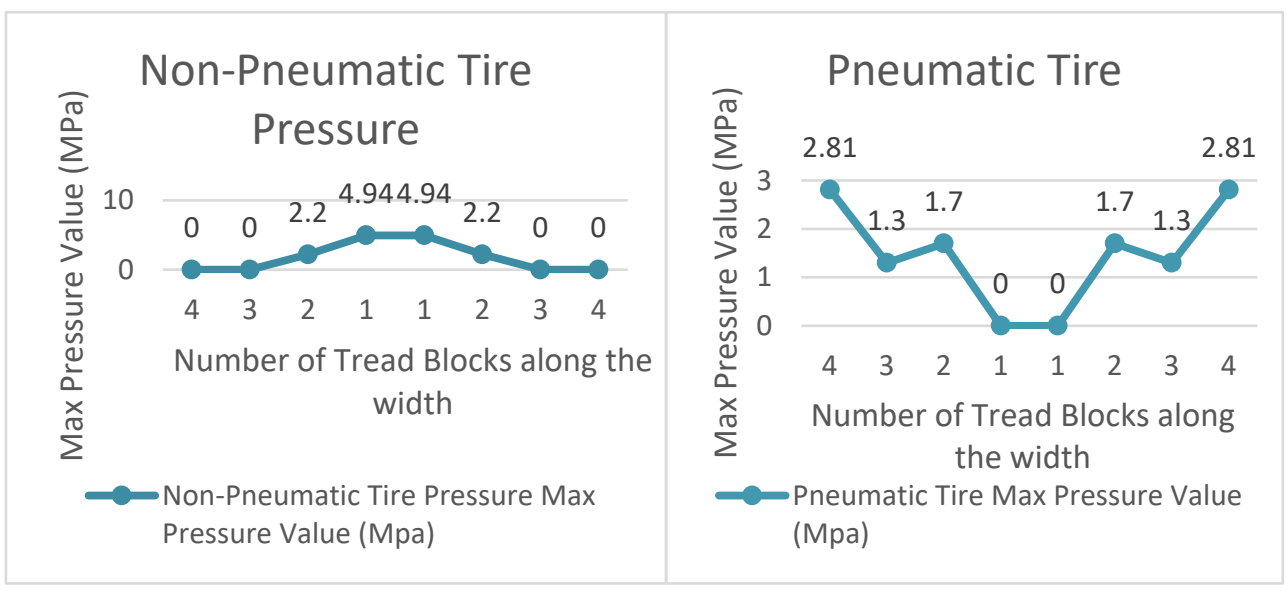

Figure 23(a): Pneumatic and Non-pneumatic pressure profile curves plotted together 


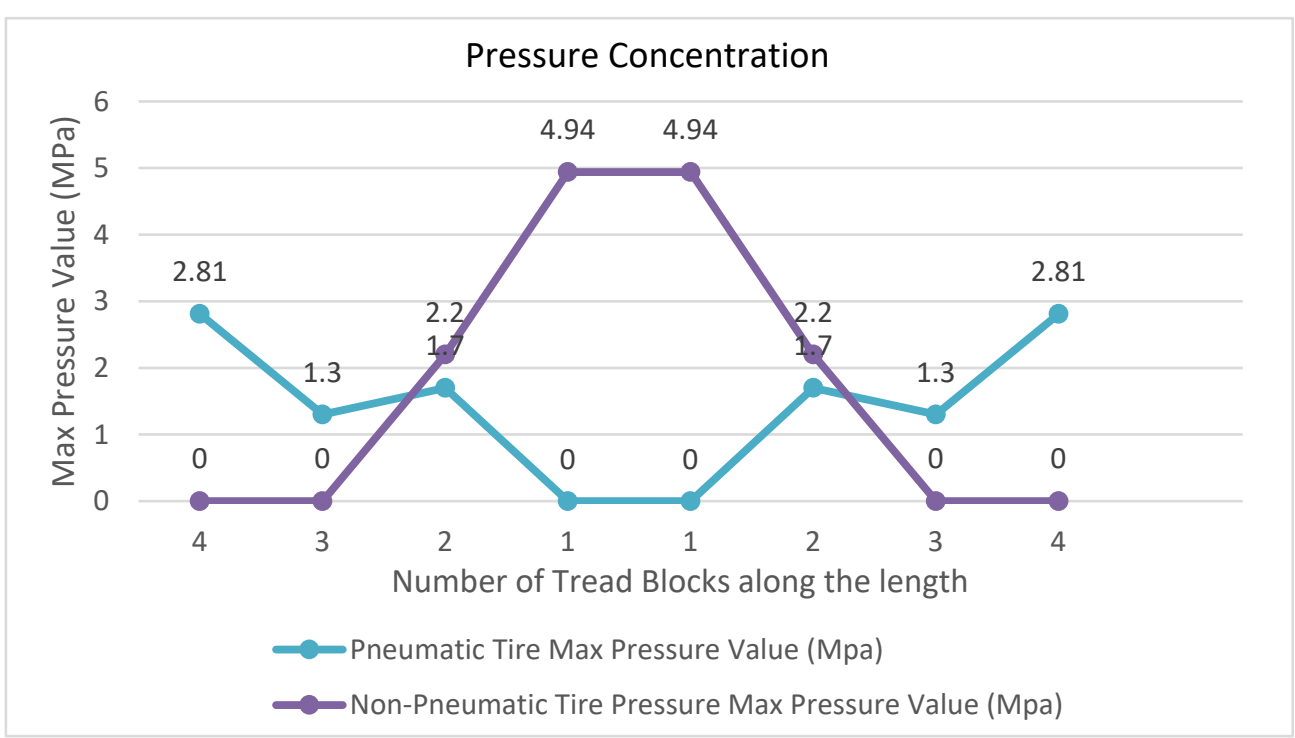

Fig 23 (b): Pneumatic and Non-pneumatic pressure profile curves plotted together

By analyzing the pressure profile curves as shown in Figure 23 (a) and (b), for pressure values across the mid-section of the tires, there is a clear trend. The pressure of the non-pneumatic model on the left is much more concentrated towards the center and has higher-pressure values. The pneumatic model's pressure concentrations cover the longitudinal length to a more considerable extent than the non-pneumatic model, with approximately four tread blocks compared to about 2 . This is a positive result as the aim of this work is to prove that the nonpneumatic tire has a more concentrated pressure profile with higher-pressure values.

\section{CONCLUSIONS}

This study confirmed that Finite Element Analysis is a valid approach to analyze the pressure profile of two kinds of tires, and to draw a comparison based on their performance in cold climatic conditions. However, for the pneumatic model, the longitudinal contact area is larger than the non-pneumatic model but the pressure on the non-pneumatic tire is more evenly distributed over the tire width, with both higher average and maximum pressure values of 4.94 MPa as compared to $2.81 \mathrm{MPa}$ for pneumatic tire. This concentration of pressure is believed to increase the grip of a tire, as the frictional force is a function of pressure. The increase in the grip of a non-pneumatic tire significantly increases the friction and reduces the chances of sliding over snow and ice in cold weather conditions. In addition, as non-pneumatic tires are based on material springs instead of air, the tire characteristics are not affected by temperature fluctuations as is the case with the air pressure inside a pneumatic tire. This research work proved that non-pneumatic tires are a better choice for use in winters. Carefully designed nonpneumatic tires will be useful in avoiding the detrimental impact loads inflicted on the roads by either studded or chained tires. 


\section{REFERENCES}

[1] T. Ahmad, T. Rashid, H. Khawaja, and M. Moatamedi, "Study of Wind Chill Factor using Infrared Imaging,” Int. J. Multiphys., vol. 10, no. 3, pp. 325 - 341, 2016.

[2] H. Xue, and H. Khawaja, "Review of the Phenomenon of Ice Shedding from Wind Turbine Blades,” Int. J. Multiphys., vol. 10, no. 3, pp. 265 - 276, 2016.

[3] M. Virk, M. Virk, U. Mughal, Q. Hu, and X. Jiang, "Multiphysics Based Numerical Study of Atmospheric Ice Accretion on a Full Scale Horizontal Axis Wind Turbine Blade,” Int. J. Multiphys., vol. 10, no. 3, pp. 237-246, 2016.

[4] H. Xue, and H. Khawaja, "Investigation of Ice-PVC separation under Flexural Loading using FEM Analysis,” Int. J. Multiphys., vol. 10, no. 3, pp. 247 - 264, 2016.

[5] T. Ahmad and H. Khawaja, "Review of low-temperature crack (LTC) developments in asphalt pavements,” Int. J. Multiphys., vol. 12, no. 2, pp. 169-187, 2018.

[6] M. J. Angerinos, R. L. Moore, J. P. Mahoney, and A. J. O. Brien, “A Synthesis on Studded Tires,” Washington State Department of Transportation Transportation Building, MS 7370 Olympia, Washington 98504-7370, 1999. [Online]. Available: https://www.wsdot.wa.gov/research/reports/fullreports/471.1.pdf.

[7] N. Fukuzaki, T. Yanaka, and Y. Urushiyama, "Effects of studded tires on roadside airborne dust pollution in Niigata, Japan,” Atmos. Environ., vol. 20, no. 2, pp. 377-386, 1986.

[8] B. Jancsek-Turóczi, A. Hoffer, I. Nyírö-Kósa, and A. Gelencsér, "Sampling and characterization of resuspended and respirable road dust,” J. Aerosol Sci., vol. 65, pp. 69-76, 2013.

[9] H. A. Khawaja and K. Parvez, "Validation of normal and frictional contact models of spherical bodies by FEM analysis,” Int. J. Multiphys., vol. 4, no. 2, pp. 175-185, 2010.

[10] A. Albers, A. Albers, B. Lorentz, and L. Nowicki, "Numerical investigations in mixed friction systems,” Int. J. Multiphys., vol. 4, no. 1, pp. 83-94, 2010

[11] S. Ludvigsen, "Improving mechanical grip on winter tires. Finite element analysis on pressure profile of airless tire compared to conventional tire using ANSYS workbench," UiT - the Arctic University of Norway, 2017.

[12] S. Ag and G. Ferrer, The Economics of Tire Remanufacturing. INSEAD, Fontainebleau, France, 1993.

[13] J. A. Davisson, “Design and Application of Commercial Type Tires,” SAE Transactions, vol. 78. SAE International, pp. 1-32, 1969.

[14] B. M. Gallaway et al., "Pavement and Geomeric Design Criteria for Minimizing Hydroplaning,” Dec. 1979.

[15] G. P. Ong and T. F. Fwa, "Wet-Pavement Hydroplaning Risk and Skid Resistance: Modeling,” J. Transp. Eng., vol. 133, no. 10, pp. 590-598, 2007.

[16] J. D. Cullen, N. Arvanitis, J. Lucas, and A. I. Al-Shamma'a, "In-field trials of a tyre pressure monitoring system based on segmented capacitance rings,” Meas. J. Int. Meas. Confed., vol. 32, no. 3, pp. 181-192, 2002. 
[17] H. V Raniecki, B; Nguyen, "Isotropic elastic-plastic solids at finite strain and arbitrary pressure,” Arch. Mech.; (Poland); J. Vol. 365-6, pp. 687-704, Accessed: Aug. 08, 2020. [Online]. Available: https://www.osti.gov/etdeweb/biblio/5726644.

[18] T. Umeno, K. Asano, H. Ohashi, M. Yonetani, T. Naitou, and T. Taguchi, "Observer based estimation of parameter variations and its application to tyre pressure diagnosis," Control Eng. Pract., vol. 9, no. 6, pp. 639-645, Jun. 2001.

[19]Z. Andleeb et al., "Multiphysics Analysis of CFRP Charpy Tests by varying Temperatures,” Int. J. Multiphys., vol. 14, no. 2, pp. 143-160, 2020.

[20] C. Strand, Z. Andleeb, and H. A. Khawaja, "Multiphysics Impact Analysis of Carbon Fiber Reinforced Polymer (CFRP) Shell," Explos. Shock Waves High Strain Rate Phenom., vol. 13, pp. 115-120, 2019.

[21]B. T. Fijalkowski and B. T. Fijalkowski, "Basics of Automotive Vehicle Braking,” in Automotive Mechatronics: Operational and Practical Issues, Springer Netherlands, 2011, pp. 435-451.

[22] J. Ju, D. M. Kim, and K. Kim, "Flexible cellular solid spokes of a non-pneumatic tire," Compos. Struct., vol. 94, no. 8, pp. 2285-2295, 2012.

[23] C. K. Lee, S. C. Tan, F. F. Wu, S. Y. R. Hui, and B. Chaudhuri, "Use of Hooke's law for stabilizing future smart grid the electric spring concept," in 2013 IEEE Energy Conversion Congress and Exposition, ECCE 2013, 2013, pp. 5253-5257.

[24] T. H. Fay and S. D. Graham, “Coupled spring equations,” Int. J. Math. Educ. Sci. Technol., vol. 34, no. 1, pp. 65-79, 2003.

[25] M. Moatamedi, H Khawaja, Finite Element Analysis. CRC Press, 2018.

[26] U. N. Mughal, H. A. Khawaja, and M. Moatamedi, "Finite element analysis of human femur bone,” Int. J. Multiphys., vol. 9, no. 2, pp. 101-108, 2015.

[27] O. Myrli and H. Khawaja, "Fluid-structure interaction (FSI) modelling of aquaculture net cage,” Int. J. Multiphys., vol. 13, no. 1, pp. 97-111, 2019.

[28] D. Brunner, H. Khawaja, M. Moatamedi, and G. Boiger, “CFD modelling of pressure and shear rate in torsionally vibrating structures using ANSYS CFX and COMSOL Multiphysics,” Int. J. Multiphys., vol. 12, no. 4, pp. 349-358, 2018.

[29] Khawaja, Raouf, Parvez, and Scherer, "Optimization of elstomeric micro-fluidic valve dimensions using non-linear finite element methods,” Int. J. Multiphys., vol. 3, no. 2, pp. 187-200, 2009.

[30] L. Ann Myers, R. Roque, B. E. Ruth, and C. Drakos, "Measurement of Contact Stresses for Different Truck Tire Types To Evaluate Their Influence on Near-Surface Cracking and Rutting,” Transp. Res. Rec. J. Transp. Res. Board, vol. 1655, no. 1, pp. 175-184, 1999.

[31] P. M. O. Owende, A. M. Hartman, S. M. Ward, M. D. Gilchrist, and M. J. O’Mahony, "Minimizing Distress on Flexible Pavements Using Variable Tire Pressure,” J. Transp. Eng., vol. 127, no. 3, pp. 254-262, 2001. 
[32] B. Clapson and G. Lake, "Truck tire groove cracking theory and practice,” Rubber Chem. Technol., vol. 44, no. 5, pp. 1186-1202, 1971.

[33] H. Khawaja, "Application of a 2-D approximation technique for solving stress analyses problem in FEM,” Int. J. Multiphys., vol. 9, no. 4, pp. 317-324, 2015.

[34] H. A. Khawaja, T. A. Bertelsen, R. Andreassen, and M. Moatamedi, "Study of CRFP Shell Structures under Dynamic Loading in Shock Tube Setup,” J. Struct., vol. 2014, pp. 1-6, 2014.

[35]E. Stange, Z. Andleeb, H. Khawaja, and M. Moatamedi, "Multiphysics study of tensile testing using infrared thermography,” Int. J. Multiphys., vol. 13, no. 2, pp. 191-202, 2019.

[36] E. Stange, Z. Andleeb, and H. A. Khawaja, "Qualitative visualization of the development of stresses through infrared thermography,” Vestn. MGTU, vol. 22, no. 4, pp. 503-507, 2019. 
